\title{
Pyramid wave-front sensing with a laser guide star for an ELT
}

\author{
Brice Le Roux ${ }^{\mathrm{a}}$ \\ Laboratoire d'Astrophysique de Marseille. Observatoire Astronomique de Marseille Provence. Uni- \\ versité de Provence
}

\begin{abstract}
We present a study of the behaviour and performance of the pyramid WFS when the guide star is a laser GS on an ELT. Several aspects of a laser based wave front sensing are considered, as the fact that the considered object is extended, but also the consequences of the width of the sodium layer on a pyramid measurement. Simulation results are presented.
\end{abstract}

\section{Introduction}

The wavefront sensor [WFS] is a key element of an Adaptive Optics [AO] system. It gives access to a direct measurement of the turbulent phase, its curvature or its slope, from which the mirror voltages are computed. The ability of the system to correct efficiently the atmospheric turbulence is strongly dependent on the performance of the WFS in estimating the turbulent phase. The Shack-Hartmann [SH] WFS has been for a long time the standard used in AO systems. In 1996, it has been proposed [1] a new generation WFS, the pyramid WFS. It is a focal plane WFS, based on the principle of a Foucault knife-edge. It has been demonstrated that it provides a consistent gain with respect to the Shack-Hartmann [2,5-7]. More recently, improvements were proposed to increase the pyramid performance [3,4]. On the framework of the developpement of extremely large telescopes, the interest for a pyramid wave front sensor appears clearly. Indeed, as the pyramid WFS is located on the focal plane, when the diameter of the telescope gets larger, the size of the image on the focal plane gets smaller (in closed loop) and therefore the sensitivity of the pyramid gets better. But its behaviour with laser guide stars, most probably necessary in any Extremely Large Telescope [ELT], is still relatively unknown. Some WFS dedicated to laser GS wave front sensing has already been proposed [8,9] but a full study of the pyramid WFS behaviour is still missing today. This work's aim is to bring first answers to this topic, based on numerical simulations of a pyramid WFS.

\section{Pyramid wave front with a laser guide star}

The pyramid WFS is based on the Foucault knife-edge effect. On the focal plane, it gives access to the phase of the incident wave front or, when the pyramid is modulated, to the first derivative of the phase, as the Shack Hartmann sensor. As the pyramid WFS is located on the focal plane, when the diameter of the telescope gets larger, the size of the image on the focal plane gets smaller (in closed loop) and therefore the sensitivity of the pyramid gets better. The modulation of the pyramid consists in moving in the focal plane the pyramid on a square or a circle. As a first approximation, one can imagine that a pyramid will behave with the modulation as a fixed pyramid in presence of a very special extended object, whose geometry is given by the modulation. For example, a circulare modulation of a pyramid is equivalent to a fixed pyramid looking to a ring, or a circle, depending on the size of the object. One can then anticipate the links between the modulation of the pyramid and the size of the object.

The use of laser guide star presents several constraints on the WFS. We will study here two of those constraints. The object is a $2 \mathrm{D}$ object, due to the propagation of the beacon inside the turbulence

\footnotetext{
a e-mail: brice.leroux@oamp.fr
} the original work is properly cited. 
the way up. The object is also in fact a 3D object due to the width of the sodium layer (we will consider only sodium lasers). On a Shack-Hartmann, this width implies the so called elongation effect. On a pyramid, as the WFS is on the focal plane, the object seen on the focal plane is elongated in the z-axis.

\section{Simulating the extended object for the pyramid}

\subsection{2-D extended object -x,y- simulation}

The circular modulation of the pyramid wave-front sensor is equivalent to a circular fast movement of the image on the focal plane. As the pyramid wave front sensor considers not only the image but the complex amplitude, the modulation of the pyramid WFS was modelized as a variating tilt added to the phase in the complex amplitude on the focal plane. This tilt variation is chosen to make the image moving on a circle on the focal plane. Finally, as the modulation is much faster than the temporal frequency of the system, an average is operated on the images behind the pyramid. The pyramid WFS considers the complex amplitude on the focal plane. It is therefore not possible to use the simple imageobject convolution relation. We chose to spatially sample the object and propagate each sampling point through the pyramid WFS. Each of these points was considered as having the same complex amplitude except for the phase. A different position on the field is a different tilt in the phase.

It is interesting to notice that the modulation of the pyramide can therefore be considered as a way to artificially obtain an extended object, with a special geometry. In the case of a circular geometry, a point source object is artificially extended as a circle by the pyramid modulation. We consider the case of a diameter such as $D / r 0=45$, a WFS with $45 \times 45$ subapertures $(45 \times 45$ pixels on the camera behind the pyramid) and we consider enough flux to ignore the noise effects. The error variance was computed for each of 100 realisations and we obtained a mean error variance in $\mathrm{x}$ and $\mathrm{y}$ and deduce the global variance of error.

The optimisation of the modulation radius was done by simulating several times the same pyramid WFS measurement on the same object and changing only the radius of modulation. There is an optimal value of the modulation radius that can be found in that operation. To simulate an extended object, we chose to sample the object and simulate $\mathrm{N}$ times a point object. Each sampling point delivers a phase screen which contains a different tip/tilt. This simulation procedure is very similar to the one applied for the modulation procedure, except that on the modulation case, the simulated points are located on a circle. The extended object we simulated is gaussian. Its size was tuned to check the performance of the pyramid on several object sizes.

\subsection{Pyramid with 2-D object simulation results}

We already said that the modulation of the pyramid has a effect comparable to a particular extended object, with a circular geometry (if the modulation is circular). We could expect that the modulation radius needed is lower in the extended object case. The first conclusion of the results presented in figure 1 and 2 is that a modulation is needed for the pyramid wave front sensor even in the case of an extended object. The optimal modulation radius is slightly smaller in the extended object case but not significantly (around 10\% smaller). The second conclusion is that the modulated pyramid WFS is able to measure the turbulent phase slopes on an extended object. Finally, figure 2 shows that when the object becomes too large, the pyramid fails in measuring correctly the slopes. In figure 2, the left part of the curve can let imagine that the pyramid does better when the object is slightly extended. In fact, this is probably due to the low precision of the modulation radius estimation in the simulations. In figure 1 , we see a quasi plateau around the optimal radius due to this.

\subsection{3-D extended object -x,y,z- simulation}

The thickness of the sodium layer create on a Shack Hartmann an elongation of the spots behind the microlenses array. On a pyramid, placed on a focal plane, the elongation is on the z-axis. The 


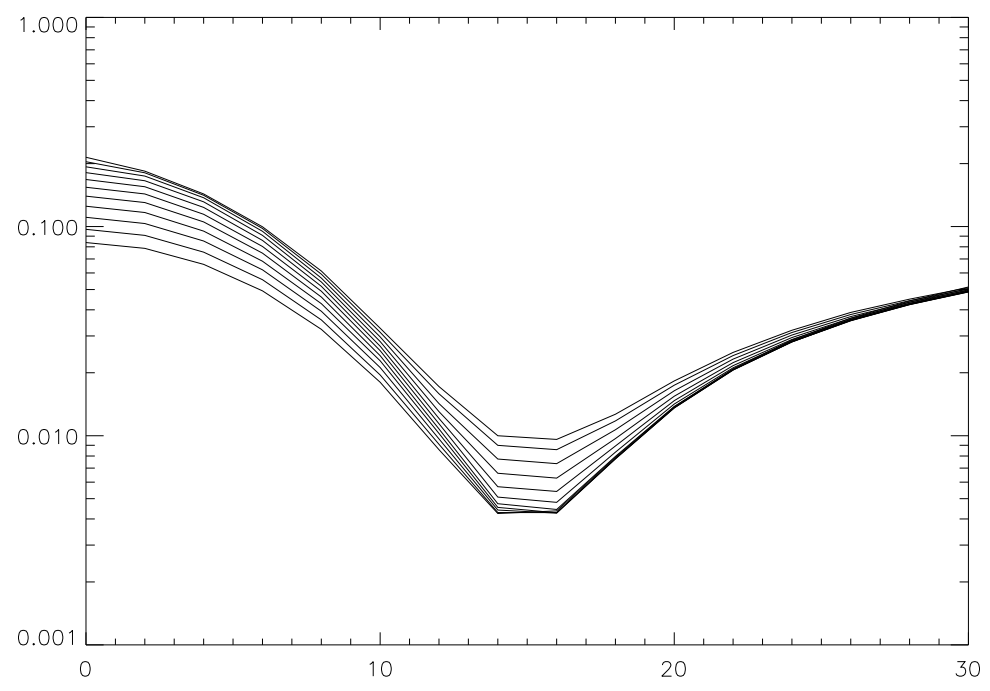

Fig. 1. Error variance (radian square) as a function of the modulation radius (in 2 lambda/D) for several size of object.

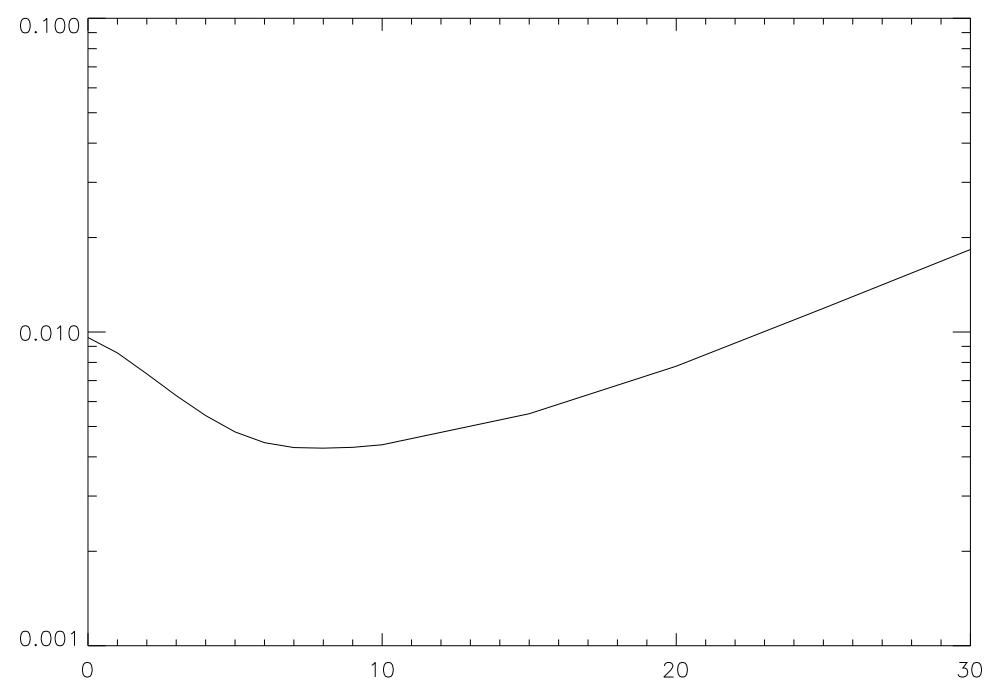

Fig. 2. Error variance (Radian square) as a function of the extended object size (in 2 lambda/D) when the modulation radius is optimized.

modelisation of a 3D x-y-z extended object allows to simulate a complete laser guide star measurement by the pyramid WFS. Simulating an object extended along the $\mathrm{z}$ axis is equivalent to simulate several $2 \mathrm{D}$ objects along the $\mathrm{z}$ axis. Those several $2 \mathrm{D}$ objects are sampling the $3 \mathrm{D}$ object. The phase screen from each of them contains a different defocalisation.

The method to simulate a $\mathrm{z}$-elongation object is similar to the method to simulate an $\mathrm{x}-\mathrm{y}$ extended object. The pyramid measurement is done for several wave fronts coming from several altitudes, therefore with a different defocus. We chose the defocus aberration range in order to be characteristic of a sodium layer altitude of $90 \mathrm{~km}$ and a thickness of the sodium layer of $5 \mathrm{~km}$. Once the measurement is 


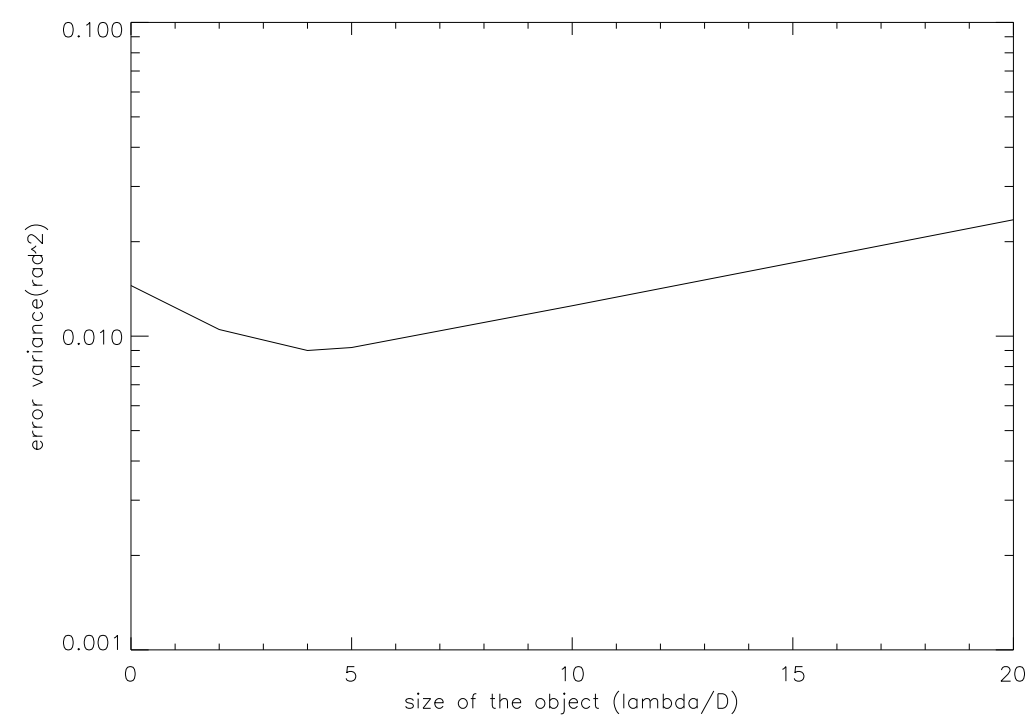

Fig. 3. Error variance (Radian square) as a function of the extended object size (in 2 lambda/D) when the modulation radius is optimized in the case of a $x-y-z$ elongation

done for several wave fronts within the defocus range, the average gives the pyramid measurement of the $3 \mathrm{D}$ object.

\subsection{Pyramid with 3-D extended object simulation results}

The simulation results are presented in figure 3. The same results than figure 3 are plot when the thickness of the sodium layer is taken into account. One can observe an important influence of the $\mathrm{z}$-axis elongation on the pyramid performance. The variance error is multiplied by a factor between 1.5 and 2 when the object become z-elongated. One could conclude from the presented performances of the pyramid WFS that the pyramid is maybe not optimal for working with a laser guide star. It might simply mean that complementary works is needed to optimize pyramid measurements of a laser guide star, as it has already been done for a long time for the Shack Hartmann WFS.

\section{Conclusion}

We have shown that the pyramid WFS is efficient with an $x, y$ extended object (2D), but it loses some performance on a 3D extended object (considering z-elongation). This allows to imagine using a pyramid WFS on a ELT Still remain to be studied other aspects of laser GS measurement as the fact that the wave is spherical. With LGS, the spot on the pyramid never becomes $\lambda / D$, because the object is extended. This brings us to the conclusion that the pyramid WFS looses its main advantage with a LGS and that it becomes equivalent to a $\mathrm{SH}$.

\section{References}

1. Ragazzoni, R., 'Pupil plane wave-front sensing with an oscillating prism,' J. of Mod. Opt., Vol. 43, 289-293, 1996.

2. Esposito, S. and Riccardi, A., A\&A, Vol.372, 710, 2001 
3. Ragazzoni, R., 'AO for ELTs. How much margin for innovation?', in Extremely large telescopes, A.L.Ardeberg, T.Andersen, eds., Proc. SPIE 5382, 456 (2003)

4. Le Roux,B., Ragazzoni,R., 'Beating the Poisson limit by coupling an occulting mask to wavefront sensing', Monthly Notices of the Royal Astronomical Society: Letters, Volume 359, Issue 1, pp. L23-L26 (2005)

5. Ragazzoni, R., Farinato, J. 'Sensitivity of a pyramidic Wave Front sensor in closed loop Adaptive Optics,' A\&A, Vol. .350, p.L23-L26, 1999.

6. Vérinaud, C., 'On the nature of the measurements provided by a pyramid wave-front sensor', Optics Communications, Vol. 233, Issue 1-3, 27-38, 2004.

7. Vérinaud, C., 'Adaptive optics for high contrast imaging: Pyramid sensor vs. spatially filtered Shack-Hartmann'Advances in Adaptive Optics II. Edited by Ellerbroek, Brent L.; Bonaccini Calia, Domenico. Proceedings of the SPIE, Volume 6272, pp. 62723Q (2006)., MNRAS, in press , 2004.

8. Meyer, E.; Gaessler, W.; Kellner, S. A.; Diolaiti, E.; Egner, S.; Ragazzoni, R.; Farinato, J., 'Multiconjugated adaptive optics for ELTs: an enhancement of the PIGS setup', Advances in Adaptive Optics II. Edited by Ellerbroek, Brent L.; Bonaccini Calia, Domenico. Proceedings of the SPIE, Volume 6272, pp. 62723Q (2006).

9. Kellner, Stephan; Ragazzoni, Roberto; Gassler, Wolfgang; Diolaiti, Emiliano; Farinato, Jacopo; Arcidiacono, Carmelo; Myers, Richard M.; Morris, Tim J.; Ghedina, Adriano, 'PIGS on sky dream or reality?', Second Backaskog Workshop on Extremely Large Telescopes. Edited by Ardeberg, Arne L.; Andersen, Torben. Proceedings of the SPIE, Volume 5382, pp. 520-525 (2004). 